\title{
EXTREMAL SOLUTIONS FOR A CLASS OF NONLINEAR DIFFERENTIAL EQUATIONS
}

\author{
KURT KREITH
}

\begin{abstract}
Positive solutions of $y^{(n)} \pm f(t, y)=0$ are characterized as maximal or minimal depending on whether their asymptotic behavior is $O\left(t^{n-1}\right)$ or $O(1)$, respectively. Necessary and sufficient conditions for the existence of such extremal solutions are established.
\end{abstract}

This paper deals with various forms of minimal and maximal asymptotic behavior for positive solutions of nonlinear equations of the form

$$
\begin{aligned}
& y^{(n)}+f(t, y)=0, \\
& y^{(n)}-f(t, y)=0,
\end{aligned}
$$

where $f(t, y)$ is continuous in $[0, \infty) \times(-\infty, \infty)$ and satisfies

$$
y f(t, y)>0 \text { whenever } y \neq 0 \text {. }
$$

Our definitions and results are strongly motivated by a recent paper of Kusano and Naito [3] which deals with super- and sublinear equations of the form

$$
\left(r(t) y^{\prime \prime}\right)^{\prime \prime}+f(t, y)=0
$$

and contains our results for the equation $\left(1^{+}\right)$when $n=4$. They are also related to oscillation theory for strongly nonlinear differential equations insofar as the existence of such extremal solutions has been used to show the necessity of various oscillation criteria. In this context variations and special cases of our results appear in [5] and [6].

Basic to the consideration of minimal solutions is the fact that because of (2), any eventually positive solution of $\left(1^{+}\right)\left[\left(1^{-}\right)\right]$will satisfy $y^{(n)}(t)<0[>0]$ for sufficiently large $t$. By successive applications of Rolle's Theorem it follows that $y^{(k)}(t)$ will eventually be of constant sign for $0<k<n$. Also, if a solution is to remain positive and bounded as $t \rightarrow \infty$, then we must eventually have

$$
y^{(k)}(t) y^{(k+1)}(t)<0 \text { for } 1<k<n-1 .
$$

These observations lead us first to consider $\left(1^{+}\right)\left[\left(1^{-}\right)\right]$when $n$ is odd [even]. It is now natural to refer to a positive solution of $\left(1^{+}\right)$or $\left(1^{-}\right)$as minimal in case $(-1)^{k} y^{(k)}(t)$ is eventually positive for $0<k<n$, and the existence of such minimal solutions follows readily from a theorem of Hartman and Wintner [1]. Here we need only impose growth conditions on $f(t, y)$ which assure that solutions of $\left(1^{+}\right)$

Received by the editors November 16, 1978 and, in revised form, July 30, 1979.

AMS (MOS) subject classifications (1970). Primary $34 \mathrm{C10}$.

Key words and phrases. Maximal solutions, minimal solutions, iteration, asymptotic behavior, critical solutions. 
and $\left(1^{-}\right)$can be continued to $t=\infty$ and consider the vector $\mathbf{x}=$ $\left(y,-y^{\prime}, y^{\prime \prime}, \ldots,(-1)^{n} y^{(n)}\right)$ which satisfies the first order system

$$
\mathbf{x}^{\prime}=-\mathbf{f}(t, \mathbf{x}) \text {. }
$$

Writing $\mathbf{v}>\mathbf{0}$ in case all components of $\mathbf{v}$ are positive, we note that in (4) $f(t, x)>0$ whenever $x>0$. According to [1] (see also [2]) this assures the existence of a "monotone solution" $\mathbf{x}(t)$ of (4) satisfying $\mathbf{x}(t)>0$ in $[0, \infty)$.

If, on the other hand, we consider $\left(1^{+}\right)\left[\left(1^{-}\right)\right]$when $n$ is even [odd], we cannot hope to obtain minimal solutions which correspond to monotone vector solutions in the sense of [1]. The only chance to obtain a bounded solution $y(t)$ occurs in case

$$
\begin{aligned}
y^{(2 k)}(t) \uparrow 0 & \text { for } 1<k \leqslant[n / 2], \\
y^{(2 k+1)}(t) \downarrow 0 & \text { for } 0<k<[n / 2],
\end{aligned}
$$

in which case it is possible that $y(t) \uparrow k$ for some $k<\infty$. Accordingly, for $n$ even [odd] we refer to solutions of $\left(1^{+}\right)\left[\left(1^{-}\right)\right]$as minimal in case they are bounded and seek growth conditions on $f(t, y)$ which assure their existence.

The growth conditions on $f(t, y)$ will be formulated in terms of a continuous function $g(t, y)$ which is monotone increasing or decreasing in $y$ and satisfies

$$
g(t, y) \geqslant f(t, y)
$$

for $(t, y) \in[0, \infty) \times[0, \infty)$. Specifically we have the following result.

THEOREM 1. Suppose that $n$ is even [odd]. A sufficient condition for $\left(1^{+}\right)\left[\left(1^{-}\right)\right]$to have a minimal (bounded) positive solution is that

$$
\int^{\infty} t^{n-1} g(t, c) d t<\infty
$$

for some constant $c>0$.

Proof. Our proof depends on the construction of a solution $Y(t)$ of $\left(1^{+}\right)\left[\left(1^{-}\right)\right]$ which is positive and "critical" in the sense that it satisfies initial conditions which make it possible to satisfy (5). It will be shown that if (7) is satisfied, then we can choose an initial $t=T$ and $Y(T)$ so that $Y(t)$ remains bounded as $t \rightarrow \infty$.

To motivate the form of this critical solution it is helpful to consider explicitly the case $n=2$ before indicating the extension to general $n$. Since for $n=2$

$$
y^{\prime}(t)=y^{\prime}(T)-\int_{T}^{t} f(s, y(s)) d s
$$

it follows that a critical solution $Y$ must satisfy $Y^{\prime}(T)=\int_{T}^{\infty} f(s, Y(s)) d s$ and

$$
Y(t)=Y(T)+(t-T) \int_{T}^{\infty} f(s, Y(s)) d s-\int_{T}^{t}(t-s) f(s, Y(s)) d s
$$

Therefore, $Y(t)=Y(T)+t \int_{t}^{\infty} f(s, Y(s)) d s-T \int_{T}^{\infty} f(s, Y(s)) d s+\int_{T}^{t} s f(s, Y(s)) d s$ and

$$
Y(t)=Y(T)+\left.F(x ; Y)\right|_{x=T} ^{x=t}+\int_{T}^{t} s f(s, Y(s)) d s
$$

where $F(x ; Y)=x \int_{x}^{\infty} f(s, Y(s)) d s<\int_{x}^{\infty} s g(s, Y(s)) d s$, so that $\lim _{x \rightarrow \infty} F(x)=0$ if $\int^{\infty} \operatorname{tg}(t, Y(t)) d t<\infty$. For $n>1$ similar considerations lead one to consider a 
critical solution defined in terms of the integral equation

$$
\begin{aligned}
Y(t)= & Y(T)+\sum_{j=0}^{n-2}(-1)^{j} \frac{(t-T)^{j+1}}{(j+1) !} \int_{T}^{\infty} \frac{(s-T)^{n-j-2}}{(n-j-2) !} f(s, Y(s)) d s \\
& -(-1)^{n} \int_{T}^{t} \frac{(t-s)^{n-1}}{(n-1) !} f(s, Y(s)) d s
\end{aligned}
$$

which reduces to $(8)$ in case $n=2$. It is clear that a solution of $\left(8^{\prime}\right)$ is a solution of $\left(1^{+}\right)\left[\left(1^{-}\right)\right]$, while differentiation of $\left(8^{\prime}\right)$ yields

$$
\begin{aligned}
Y^{\prime}(t)= & \int_{T}^{\infty} \sum_{j=0}^{n-2} \frac{(T-t)^{j}}{j !} \frac{(s-T)^{n-j-2}}{(n-j-2) !} f(s, Y(s)) d s \\
& -(-1)^{n} \int_{T}^{t} \frac{(t-s)^{n-2}}{(n-2) !} f(s, Y(s)) d s
\end{aligned}
$$

or, after some manipulation using the binomial expansion for $(t-s)^{n-2}=$ $[(t-T)-(s-T)]^{n-2}$, we get

$$
Y^{\prime}(t)=(-1)^{n} \int_{t}^{\infty} \frac{(t-s)^{n-2}}{(n-2) !} f(s, Y(s)) d s
$$

and

$$
Y^{(k)}(t)=(-1)^{n} \int_{t}^{\infty} \frac{(t-s)^{n-k-1}}{(n-k-1) !} f(s, Y(s)) d s \quad \text { for } 1<k<n-1 .
$$

In order to get an expression for $Y(t)$ corresponding to (9), we integrate the last equation for $Y^{\prime}(t)$ to get

$$
\begin{aligned}
Y(t)= & Y(T)+(-1)^{n} \int_{T}^{t} \int_{\tau}^{\infty} \frac{(\tau-s)^{n-2}}{(n-2) !} f(s, Y(s)) d s d \tau \\
= & Y(T)+(-1)^{n} \int_{T}^{t} f(s, Y(s)) \int_{T}^{s} \frac{(\tau-s)^{n-2}}{(n-2) !} d \tau d s \\
& +(-1)^{n} \int_{t}^{\infty} f(s, Y(s)) \int_{T}^{t} \frac{(\tau-s)^{n-2}}{(n-2) !} d \tau d s \\
= & Y(T)-(-1)^{n} \int_{T}^{t} \frac{(T-s)^{n-1}}{(n-1) !} f(s, Y(s)) d s \\
& +(-1)^{n} \int_{t}^{\infty}\left[\frac{(t-s)^{n-1}}{(n-1) !}-\frac{(T-s)^{n-1}}{(n-1) !}\right] f(s, Y(s)) d s \\
= & Y(T)-(-1)^{n} \int_{T}^{\infty} \frac{(T-s)^{n-1}}{(n-1) !} f(s, Y(s)) d s \\
& +(-1)^{n} \int_{t}^{\infty} \frac{(t-s)^{n-1}}{(n-1) !} f(s, Y(s)) d s .
\end{aligned}
$$


Separating off the $k=0$ term in the binomial expansion of the above integrands, we finally obtain

$$
Y(t)=Y(T)+\left.F(x ; Y)\right|_{x=T} ^{x=t}+\int_{T}^{t} s^{n-1} f(s, Y(s)) d s
$$

where

$$
F(x ; Y)=(-1)^{n} \int_{x}^{\infty} \sum_{k=1}^{n-1} \frac{x^{k}(-s)^{n-k-1}}{k !(n-k-1) !} f(s, Y(s)) d s
$$

and $\lim _{x \rightarrow \infty} F(x)=0$ if $\int^{\infty} t^{n-1} g(t, Y(t)) d t<\infty$. Since $g(t, y)$ satisfies (7) for some $c>0$, we can choose $T$ sufficiently large so that $\int_{T}^{\infty} t^{n-1} g(t, c) d t<c / 8$ and $|F(T ; c)|<c / 8$.

Defining $a=c / 2$ in case $g(t, y)$ is nondecreasing in $y$ and $a=c$ in case $g(t, y)$ is nonincreasing in $y$, we consider the operator $\Psi$ defined by

$$
\Psi y(t)=\frac{3}{2} a+\left.F(x ; y)\right|_{x=T} ^{x=t}+\int_{T}^{t} s^{n-1} f(s, y(s)) d s
$$

and establish the existence of a bounded solution of $\left(9^{\prime}\right)$ by showing that $\Psi$ satisfies the hypotheses of the Schauder fixed point theorem. Choosing $X$ to be the Banach space $C[T, \infty)$ with sup norm, consider the closed, convex, bounded subset

$$
X_{a}=\{y \in C[T, \infty): a<y(t)<2 a \text { for } t>T\}
$$

Then our choice of $T$ assures that $\Psi$ maps $X_{a}$ into $X_{a}$, and it follows readily from the continuity of $f(t, y)$ that $\Psi$ is continuous in the norm of $X$. Thus it remains to show that $\Psi X_{a}$ is conditionally compact in the sense that $\left\{\Psi y: y \in X_{a}\right\}$ is equicontinuous on $[T, \infty)$.

From the definition of $\Psi$ we have

$$
\Psi y\left(t_{2}\right)-\Psi y\left(t_{1}\right)=\left.F(x ; y)\right|_{x=t_{1}} ^{x=t_{2}}+\int_{t_{1}}^{t_{2}} s^{n-1} f(s, y(s)) d s
$$

and

$$
\left|\Psi y\left(t_{2}\right)-\Psi y\left(t_{1}\right)\right|<|F(x ; y)|_{x=t_{1}}^{x=\infty} \mid+\int_{t_{1}}^{\infty} s^{n-1} g(s, y(s)) d s
$$

Because of (7) there exists a $\tilde{T}$ such that $\tilde{T}<t_{1}<t_{2}$ implies that $\left|\Psi y\left(t_{2}\right)-\Psi y\left(t_{1}\right)\right|$ $<\varepsilon$ for any preassigned $\varepsilon>0$ and all $y \in X_{a}$.

From the definition of $\Psi$ and $F$ it also follows that

$$
\begin{aligned}
\left|\Psi y\left(t_{2}\right)-\Psi y\left(t_{1}\right)\right|< & \sum_{k=1}^{n-1}\left(t_{2}^{k}-t_{1}^{k}\right) \int_{t_{2}}^{\infty} \frac{s^{n-k-1}}{k !(n-k-1) !} f(s, y(s)) d s \\
& +\sum_{k=1}^{n-1} t_{1}^{k} \int_{t_{1}}^{t_{2}} \frac{s^{n-k-1}}{k !(n-k-1) !} f(s, y(s)) d s \\
& +\int_{t_{1}}^{t_{2}} s^{n-1} f(s, y(s)) d s .
\end{aligned}
$$

Since (7) assures that the first integral above is convergent uniformly for $y \in X_{a}$, one can find $\delta>0$ so that $\left|t_{1}-t_{2}\right|<\delta$ and $t_{1}<t_{2}<\tilde{T}$ imply that $\mid \Psi y\left(t_{2}\right)-$ $\Psi y\left(t_{1}\right) \mid<\varepsilon$. Thus we can decompose $[T, \infty)$ into a finite number of subintervals in 
each of which $\Psi y(t)$ has oscillation less than $\varepsilon$. By invoking the criterion for equicontinuity of Levitan $[4,83]$ which was used in [3], we conclude that $\overline{\Psi X_{a}}$ is compact, which completes the proof.

In case $f$ itself is monotone increasing or decreasing in $y$, the techniques of [3] show that the hypotheses of Theorem 1 are necessary as well as sufficient. For setting $f=g$ we consider the identity

$$
\begin{aligned}
\int_{T}^{t} \frac{s^{n-1}}{(n-1) !} f(s, y(s)) d s & =-(-1)^{n} \int_{T}^{t} \frac{s^{n-1}}{(n-1) !} y^{(n)}(s) d s \\
& =\left.(-1)^{n} \sum_{k=1}^{n}(-1)^{k} \frac{s^{n-k}}{(n-k) !} y^{(n-k)}\right|_{T} ^{t} .
\end{aligned}
$$

For a minimal solution satisfying (5) we must therefore have

$$
\int_{T}^{\infty} \frac{s^{n-1}}{n !} g(s, y(s)) d s<K+\lim _{t \rightarrow \infty} y(t)<\infty
$$

where $K$ is a constant whose value depends on $T$.

Therefore, if $a_{1}<y(t)<a_{2}$ for $t>T$, then $\int^{\infty} t^{n-1} g\left(t, a_{1}\right) d t<\infty$ if $g(t, y)$ is nondecreasing in $y$, while $\int^{\infty} t^{n-1} g\left(t, a_{2}\right) d t<\infty$ if $g(t, y)$ is nonincreasing in $y$.

Turning now to the concept of maximal solutions, we note that even in the linear case of $y^{(n)}-p(t) y=0$ we would not expect to be able to bound the growth of solutions if $p(t)>0$. Accordingly we restrict our considerations of maximal solutions to $\left(1^{+}\right)$. As observed by Kusano and Naito [3], a positive solution of (3) always satisfies $\left.\left(r(t) y^{\prime \prime}\right)\right)^{\prime \prime}<0$ and therefore cannot grow faster than

$$
\int(t(t-s) / r(s)) d s \quad \text { as } t \rightarrow \infty .
$$

Motivated by [3] we define $R(t)=t^{n-1} /(n-1)$ ! and define a positive solution $y(t)$ of $\left(1^{+}\right)$to be maximal if it is asymptotic to $c R(t)$ for some $c>0$-i.e. if there exists a constant $c>0$ such that $\lim _{t \rightarrow \infty} y(t) / t^{n-1}=c$. Our basic result regarding the existence of maximal solutions is the following:

THEOREM 2. A sufficient condition for $\left(1^{+}\right)$to have a maximal positive solution is that

$$
\int^{\infty} g(t, c R(t)) d t<\infty
$$

for some constant $c>0$.

Proof. Suppose first that $g(t, y)$ is nondecreasing in $y$ and choose $T>0$ sufficiently large so that

$$
\int_{T}^{\infty} g(t, c R(t)) d t<\frac{c}{4} .
$$

If $Y(t)$ is the solution of (1) determined by the initial conditions

$$
Y(T)=\cdots=Y^{(n-2)}(T)=0 ; \quad Y^{(n-1)}(T)=c,
$$

then $Y(t)<c(t-T)^{n-1} /(n-1) !<c R(t)$ as long as $Y(t)$ remains positive. Thus there exists a maximal interval $(T, \tilde{T})$ in which 
(i) $0<Y(t)<c R(t)$ and

(ii) $Y^{(n-1)}(t)>c / 2$.

However it follows that $\tilde{T}=\infty$, for otherwise we could use (i), (10), and the fact that $g(t, y)$ is nondecreasing in $y$ to conclude that

$$
\begin{aligned}
Y^{(n-1)}(\tilde{T}) & >Y^{(n-1)}(T)-\int_{T}^{\tilde{T}} g(s, Y(s)) d s \\
& \geqslant c-\int_{T}^{\tilde{T}} g(s, c R(s)) d s \geqslant \frac{3}{4} c .
\end{aligned}
$$

Since $Y^{(n-1)}(t)$ is decreasing in $(T, \tilde{T})$, it would follow that (i) and (ii) are satisfied in $(T, \tilde{T}]$ as well as $(T, \tilde{T})$, contradicting the maximality of $\tilde{T}$. This shows that $\tilde{T}=\infty$ and that $Y^{n-1}(t)>c / 2$ for $T<t<\infty$. Therefore,

$$
Y(t)>(c / 2)(t-T)^{n-1} /(n-1) ! \text { for } T<t<\infty,
$$

from which it follows easily that $Y(t) \sim a R(t)$ for some constant $a$ satisfying $c / 2<a<c$.

In case $g(t, y)$ is nonincreasing in $y$ we choose $T$ sufficiently large so that

$$
\int_{T}^{\infty} g(t, c R(t)) d t<\frac{1}{4}
$$

and consider the solution $Y(t)$ of $\left(1^{+}\right)$satisfying initial conditions

$$
Y^{(k)}(T)=\left(c+\frac{1}{2}\right) R^{(k)}(T) \quad \text { for } k=0, \ldots, n-2 ; \quad Y^{(n-1)}(T)=c+1 .
$$

Then for $t>T$ we have $Y(t)>\left(c+\frac{1}{2}\right) R(t)$ as long as $Y^{(n-1)}(t)>c+\frac{1}{2}$. Again letting $(T, \tilde{T})$ denote the maximal interval in which

(i) $Y(t)>\left(c+\frac{1}{2}\right) R(t)$ and

(ii) $Y^{(n-1)}(t)>c+\frac{1}{2}$

we must have $\tilde{T}=\infty$. For otherwise

$$
\begin{aligned}
Y^{(n-1)}(\tilde{T}) & \geqslant Y^{(n-1)}(T)-\int_{T}^{\tilde{T}} g(s, Y(s)) d s \\
& \geqslant c+1-\int_{T}^{\tilde{T}} g(s, c R(s)) d s>c+\frac{3}{4},
\end{aligned}
$$

so that $Y^{(n-1)}(t) \geqslant c+\frac{3}{4}$ and $Y(t)>\left(c+\frac{1}{2}\right) R(t)$ in $(T, \tilde{T}]$. It again follows that $\tilde{T}=\infty$ and that $Y(t) \sim a R(t)$ for some constant $a$ satisfying $c+\frac{1}{2}<a<c+1$.

Again, if $f(t, y)$ itself is monotone increasing or decreasing in $y$, the techniques of [3] show that the hypotheses of Theorem 2 are necessary as well as sufficient. For considering a maximal solution $y(t)$ of $y^{(n)}+g(t, y)=0$, suppose there exist positive numbers $a_{1}, a_{2}$, and $T$ such that $a_{1} R(t)<y(t)<a_{2} R(t)$ for $t>T$. Since $y(t)>a_{1} R(t)$ and $y^{(n)}(t)<0$, we must have $y^{(n-1)}(t)$ eventually positive. Integrating $\left(1^{+}\right)$yields

$$
y^{(n-1)}(t)=y^{(n-1)}(T)-\int_{T}^{t} g(s, y(s)) d s=0,
$$

so that the eventual positivity of $y^{(n-1)}(t)$ requires that $\int_{T}^{\infty} g(t, y(t)) d t<\infty$. Therefore,

$$
\int_{T}^{\infty} g\left(t, a_{1} R(t)\right) d t<\infty
$$


if $g(t, y)$ is nondecreasing in $y$, while

$$
\int_{T}^{\infty} g\left(r, a_{2} R(t)\right) d t<\infty
$$

if $g$ is nonincreasing in $y$.

\section{REFERENCES}

1. P. Hartman and A. Wintner, On monotone solutions of systems of non-linear differential equations, Amer. J. Math. 77 (1955), 860-866.

2. K. Kreith, Nonlinear differential equations with monotone solutions, Pacific J. Math. 81 (1979), 101-111.

3. T. Kusano and M. Naito, Nonlinear ascillation of fourth order differential equations, Canad. J. Math. 28 (1976), 840-852.

4. B. M. Levitan, Some problems of the theory of almost periodic functions. I, Uspehi Mat. Nauk 2 (1947), no. 5 (21), 133-192.

5. H. Onose, Oscillatory property of ordinary differential equations of arbitrary order, J. Differential Equations 7 (1970), 454-458.

6. G. Ryder and D. Wend, Oscillation of solutions of certain ordinary differential equations of nth order, Proc. Amer. Math. Soc. 25 (1970), 463-469.

Department of Mathematics, University of California, Davis, California 95616 